\title{
The predominant cultivable dental plaque flora of beagle dogs with gingivitis
}

\author{
S. A. Syed, M. Svanberg and G. Svanberg \\ Department of Oral Biology, Dental Research Institute, School of Dentistry, \\ The University of Michigan, Ann Arbor, Michigan, U.S.A. .
}

\begin{abstract}
The predominant dental plaque flora of 15 female beagle dogs (1,3 and 6 year old) with naturally developed gingivitis was studied using a continuous anaerobic culturing technique. Supra- and subgingival plaque samples from the buccal aspect of the upper third premolar were cultured on various growth media and the organisms were partially characterized. The flora in all dogs was composed mostly of anaerobic Gram negative organisms. $B$. asaccharolyticus ( $B$. melaninogenicus ss. asaccharolyticus) was found in both types of plaques in all animals and decomposed hydrogen peroxide suggesting catalase activity. $F$. nucleatum was found in higher proportions in the subgingival plaque as compared to B. asaccharolyticus and actinomycetes. Spirochetes were found in 10 of 15 supra- and in 1 of 15 subgingival specimens. S. mutans, S. mitior, S. salivarius, Veillonella, Selenomonas or vibrios could not be detected in any of the plaque samples. A combined data analysis showed, that the total viable CFU and the proportions of Gram positive organisms were significantly higher in the supra- than the subgingival plaque. Although the proportions of Gram negative bacteria were higher in subgingival plaque, the differences between the two types of plaques excluding $F$. nucleatum were not significant.
\end{abstract}

(Accepted for publication March 14, 1979)

\section{Introduction}

The beagle dog has been used in many clinical and histopathological studies on periodontal disease (Saxe et al. 1967, Hamp, Lindhe \& Heyden 1972, Hamp, Lindhe \& Löe 1973, Schroeder, Graf-DeBeer \& Attström 1975, Schroeder \& Lindhe 1975). In these studies, the severity of the disease was related to the estimated amount of microbial plaque present on the tooth surfaces and no attention was given to the composition of the dental plaque flora and its significance for the development of gingivitis and periodontitis.

Our present knowledge of the dental plaque flora in dogs is based mostly on morphologic descriptions of a few organisms in smears of pooled plaque and of plaque in situ (Krasse \& Brill 1960, Soames \& Davies 1975), studies on the aerobic/facultative flora of pooled plaque (Saphir \& Carter 1976, Wunder et al. 1976) and one report on periodontal pocket flora (Newman et al. 1977). With the exception of the last mentioned report, most of the anaerobic organisms which comprise the major component of dental plaque were not isolated, characterized or studied in great detail (Courant et al. 1968).

Therefore, it would seem necessary to examine the dominant cultivable microbial flora of beagle dogs before attempting to 
consider the etiologic role of these organisms in regard to gingival inflammation and tissue breakdown in periodontal disease. Also, such information would be helpful in establishing whether the dental plaque flora in the beagle is similar to or different from the human dental plaque flora, so as to evaluate the suitability of the beagle model for studies of periodontal disease.

The purpose of the present investigation was to study the predominant flora of supra- and subgingival plaque in adult beagles with gingivitis, but no loss of connective tissue attachment, by using quantitative anaerobic culturing procedures.

\section{Materials and Methods}

The dogs were pure-bred beagles which were raised for medical research purposes at the Laboratory Research Enterprises, Inc., Kalamazoo, Michigan. The dogs had been vaccinated for distemper and hepatitis, were regularly inspected by a veterinarian, and housed in groups of two or three in stainless steel cages. They were fed a standard pellet dog food (Bench and Field $26^{\circledR}$, Martin Feeds, New Paris, Indiana) and water ad libitum. The animals had never been on any plaque control program. Three hundred female dogs were screened and fifteen animals were selected on the basis of criteria such as age, gingival conditions and connective tissue attachment level. Three groups of animals were formed on the basis of age (one, three and six years) and equal numbers of dogs assigned to each group. Gingival Index, GI (Löe \& Silness 1963) and Plaque Index, PII (Silness \& Löe 1964) were scored on the buccal aspect of the canine as well as the mesial and distal roots of all premolars and first molars of all four quadrants.

\section{Plaque Collection}

The animals were anesthetized by intra- venous injection of Pentothal ${ }^{\circledR}$ (Abbott, North Chicago, Ill.) and two types of plaque samples, i.e. supragingival and subgingival plaque, were taken from the upper left third premolar in each animal. Supragingival plaque was collected from a two millimeter wide zone adjacent to the gingival margin at the distal root with a sterile stainless steel spoon $(1.7 \times 15 \mathrm{~mm})$ held in a hemostat. Immediately after sampling, the plaque spoon was placed into a screw cap vial filled to the rim with prereduced transport fluid (RTF, Syed \& Loesche 1972). Since the vial was completely filled with RTF, the exposure of the plaque organisms to atmospheric oxygen was minimal. The vials containing the plaque specimens were immediately introduced into an anaerobic glove box (Aranki et al. 1969) manufactured by Coy Laboratory Products (Ann Arbor, Michigan). The gaseous atmosphere of the anaerobic glove box was $85 \%$ nitrogen, $10 \%$ hydrogen, and $5 \%$ carbon dioxide.

Prior to the collection of subgingival plaque the tooth surface coronal to the gingival crevice was debrided of soft and hard deposits with a curette moved in a distomesial and slightly coronal direction. Subgingival plaque was collected with a sterile stainless steel wire loop (wire diameter, 0.3 $\mathrm{mm}$; loop diameter, $1.5 \mathrm{~mm}$ ) held in a hemostat. The loop was introduced into the crevice at a slight angle to the tooth surface without making contact with the supragingival tooth surface and moved apically until slight resistance was met. The loop was then moved back and forth in a mesiodistal direction with light pressure toward the tooth surface in order to free any adherent plaque. The loop containing the plaque was immediately placed into RTF and taken into the anaerobic glove box.

Primary Culture Media

The agar media used for primary culturing 
of the plaque specimens were: 1) Schaedler agar (Baltimore Biological Laboratories: BBL, Cockeysville, Md.), supplemented with menadione $(1 \mu \mathrm{g} / \mathrm{ml})$, dithiothreitol $(0.02 \%)$ and defibrinated sheep blood (3\%). 2) Enriched Trypticase Soy Agar (ETSA) with or without $5 \%$ sucrose. This medium contained the following: Trypticase soy agar (BBL, $4 \%$ ), agar (Difco, $0.4 \%$ ), yeast extract (Difco, 0.1\%), glucose $(0.1 \%)$, potassium nitrate $(0.05 \%)$, sodium lactate $(0.06 \%)$, sodium succinate $(0.05 \%)$, sodium formate $(0.05 \%)$, sodium fumerate $(0.1 \%)$, hemin $(1 \mu \mathrm{g} / \mathrm{ml})$, menadione $(1$ $\mu \mathrm{g} / \mathrm{ml})$, sodium carbonate $(0.04 \%)$, dithiothreitol $(0.01 \%)$, cysteine $(0.04 \%)$ defibrinated sheep blood $(3 \%)$, distilled water $(970 \mathrm{ml})$. The ETSA supplemented with sucrose was used to detect the extracellular polysaccharide forming organisms in the plaque samples.

\section{Plaque Culturing Procedures}

Inside the anaerobic glove box each plaque specimen was dispersed for 20 seconds with an ultrasonic disrupter (Kontes, Vineland, N.J.) followed by Vortex mixing for 15 seconds. Serial, five-fold dilutions in RTF were prepared and $50 \mu \mathrm{l}$ aliquots from the three last dilutions were dispensed with an Eppendorff pipette on duplicate plates of the following prereduced agar media and spread with sterile L-shaped glass rods: ETSA, ETSA with $5 \%$ sucrose and Schaedler agar. The inoculated plates were incubated in the glove box at $37^{\circ} \mathrm{C}$ for 6 to 8 days. A duplicate set of ETSA plates for each specimen was incubated aerobically to determine the aerobic colony forming units (CFU). One ETSA plate from each specimen showing 75 to 150 well isolated CFU was kept in the anaerobic glove box and used for isolation and subculturing of the organisms. The remaining plates were used for enumeration of the total and differential CFU and subsequently discarded.
Plaque protein determination and spirochete counts: The protein concentration of each plaque sample was determined by fluorometric assay (Weigele et al. 1972, Minah \& Loesche 1977) using a Turner Fluorometer (Model 111). The protein concentration of each plaque sample was used as a reference value for the total CFU. Spirochete counts were determined microscopically using the Petroff Hausser Chamber (PHC) technique (Aranki et al. 1969). Since the samples were small, the whole chamber (400 squares) was used to determine the spirochete counts.

\section{Biopsy Procedures}

Specimens comprising the buccal part of the tooth, gingiva and marginal part of the alveolar bone were taken at the plaque sampling site using the method described by Schroeder et al. (1973). After fixation in $5 \%$ glutaraldehyde and $4 \%$ paraformaldehyde at pH 7.3 (Karnovsky 1965) and decalcification in formic acid-citrate (Luna 1968) the specimens were dehydrated and embedded in Epon (Luft 1961). Buccolingual sections were prepared with an ultramicrotome (Reichert OM-U2) set at 2 microns and stained with methylene blue and basic fuchsin. Tracings of the sections were made by means of microscopic projection (linear magnification $55 \mathrm{x}$ ) and used for measuring sulcus depth and connective tissue attachment level.

\section{Isolation and Characterization of Plaque Organisms}

Isolation of the plaque organisms were made inside the anaerobic glove box using a stereo microscope with a zoom lens. Each CFU was picked and subcultured in prereduced basal esculin nitrate broth (Syed \& Loesche 1978a), incubated for 5 to 7 days and used as inoculum for various media viz. starch agar, gelatin agar (Syed 1976) and anaerobic glucose broth. The broth cul- 
tures were also used for gram stain, catalase, indole production, nitrate reduction, esculin hydrolysis and aerobic sensitivity tests. All tests were performed according to the procedures recommended in the Anaerobe Laboratory Manual (Holdeman \& Moore 1975). Whenever necessary, organic acid analysis of the glucose broth cultures was done by gas liquid chromatographic technique (Holdeman \& Moore 1975). A gas chromatograph (Varian, Palo Alto, Cal., Model 2740) equipped with a flame ionization detector and a stainless steel column (6 ft. $\times 1 / 4^{\prime \prime}$ ) containing $15 \%$ FFAP on chromsorb W (DMCS) treated) and $3 \%$ phosphoric acid (Varian, Palo Alto, Cal.) was used. The results obtained from the various tests were used to classify the organisms according to the eighth edition of Bergey's Manual of Determinative Bacteriology (1974), the Anaerobe Laboratory Manual (Holdeman \& Moore 1975) and a taxonomic scheme (Syed \& Loesche 1978a) which would facilitate the identification of various organisms in the plaque.

Streptococci were differentiated on the basis of their ability to grow on bile-esculin agar (Difco Laboratories U.S.A.), starch and esculin hydrolysis and oxygen sensitivity tests. All Gram positive, pleomorphic, non-sporing, non-motile rods, having the tendency to lose the primary stain upon decolorization with alcohol (Gram variable), arranged in $\mathrm{X}, \mathrm{Y}, \mathrm{L}$, or similar microscopic clusters, exhibiting branching upon microscopic examination were considered as actinomycetes. Those organisms which were uniformly negative for indole and gelatinase tests, and produced acetate, lactate and succinate as metabolic products were identified as Actinomyces species. Further identification of these organisms at species level was based on selected physiological and biochemical criteria including catalase, nitrate reduction, starch hydrolysis, esculin hydrolysis and oxygen sensitivity tests. If two or more physiological tests were not done due to cultivation difficulty, poor growth, loss of cultures, or the characteristics of the isolates did not fit into the species identification scheme (Syed \& Loesche 1978a) the organisms were grouped together as unidentified actinomycetes. Organisms which produced catalase, reduced nitrate and gave variable esculin hydrolysis results were grouped as $A$. viscosus. Catalase negative, esculin hydrolysis positive, facultative actinomycetes were identified as $A$. naeslundii. Organisms which hydrolyzed esculin, reduced nitrate, were negative for starch hydrolysis test and did not grow aerobically were classified as $A$. israelii. Their identification was facilitated by the white circular, cotton ball like colonies. Actinomycetes which did not reduce nitrate, but hydrolyzed starch and esculin were called $A$. bovis. Anaerobic or facultative organisms capable of producing brown or pink pigment, which reduced nitrate and gave variable esculin hydrolysis test were considered $A$. odontolyticus.

Propionibacterium acnes was identified on the basis of its characteristic microscopic morphology, and the positive catalase, indole, gelatinase and nitrate reduction tests. Gram positive, long filamentous, non-fermentative, anaerobic non-sporing rods with or without cross septa were differentiated on the basis of esculin hydrolysis tests. Since most of the physiological tests were negative, they were not identified at genus or species level.

Reddish brown or black pigmented colonies of anaerobic Gram negative pleomorphic non-motile coccobacilli which did not ferment carbohydrates, did not reduce nitrate or hydrolyse esculin, but did produce indole and gelatinase, were identified as $B$. asaccharolyticus (B. melaninogenicus ss. asaccharolyticus, Finegold \& Barnes 1977). Fusobacterium nucleatum was identified on the basis of its characteristic colonies, fusi- 
form morphology and indole positive tests and its ability to form butyric acid in the broth media. Another group of anaerobic non-motile, non-pigmented coccobacilli exhibiting agar corroding characteristics were found in the plaques. They were differentiated from pigmented bacteroides by their hazy colonies and catalase test. Since they presented considerable cultivation problems, urease test and formate-fumerate energy metabolism study were not done.

\section{Statistical Analysis}

Statistical analyses of the data were performed on the Michigan Interactive Data Analyses System (MIDAS). When comparing the different age groups the Median Test was used for analyzing ordinal level measurements (Plaque Index, Gingival Index) and Student's t-test was used for analyzing ratio level measurements (histologic measurements, bacterial counts). Comparisons between supragingival and subgingival plaque parameters were done by means of a paired t-test.

\section{Results}

\section{Clinical Parameters}

All dogs had visible plaque on most tooth surfaces and displayed clinical signs of gingival inflammation at all plaque sampling sites. There was, however, a significant difference $(p<0.05)$ in the distribution of Plaque and Gingival Index scores between the one-year-old dogs and the older animals (Figs. $1 \& 2$ ). Thus, there was a decrease in Pl.I. scores of zero and one, and an increase in Pl.I. scores of three with age. Whereas the young animals had similar numbers of GI scores of one and two, the older dogs had predominantly GI scores of two.

\section{Histologic Parameters}

As assessed in sections of specimens taken at the plaque sampling sites the mean sulcus depth was similar in all age groups and ranged from $1.02 \mathrm{~mm}$ to $1.50 \mathrm{~mm}$ (Table 1). Whereas the one-year-old dogs had no apical migration of the junctional epithelium, the older animals showed a slight but insignificant loss of connective tissue attachment (Table 1).

\section{Plaque Parameters}

Protein concentration, prevalence of spirochetes, anaerobic/aerobic ratio (all CFU recovered anaerobically/all CFU recovered aerobically) and viable CFU per sample were determined for supra- and subgingival

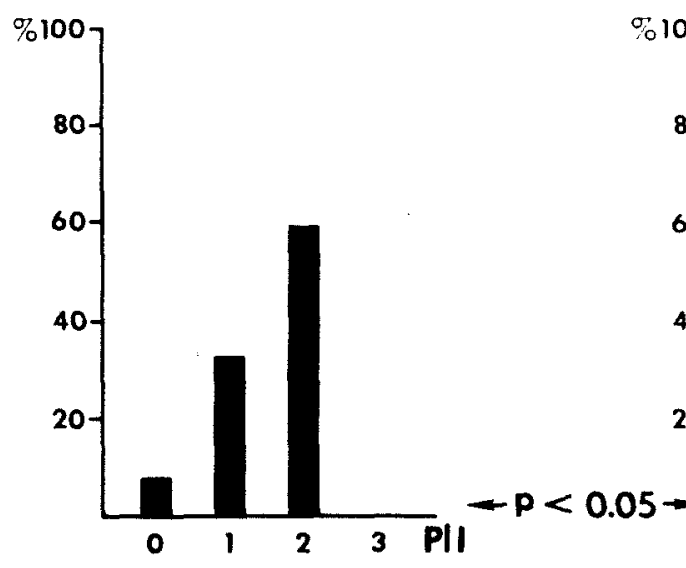

1 YEAR

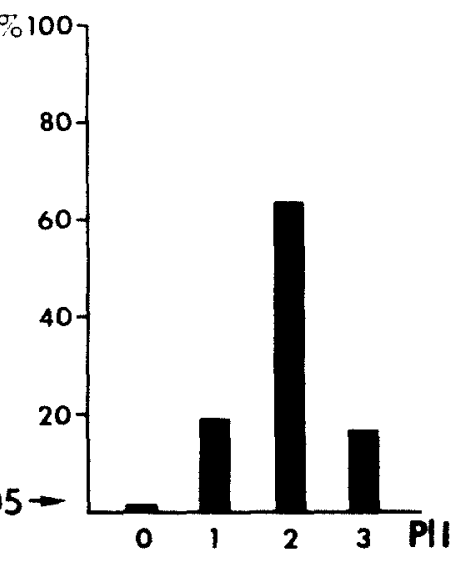

3 YEARS

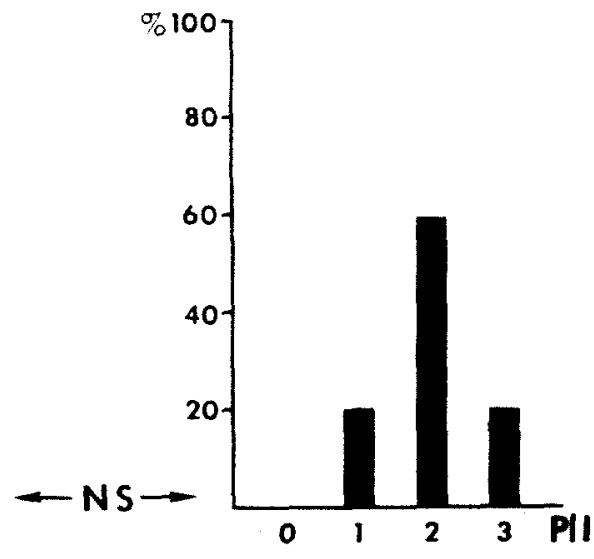

6 YEARS

Fig. 1. Distribution of Plaque Index Scores in Beagle Dogs with Gingivitis*

* Scores were assessed in canines through first molars (C1-M1) of all four quadrants and analyzed by the Median Test. Five animals in each group were used. 


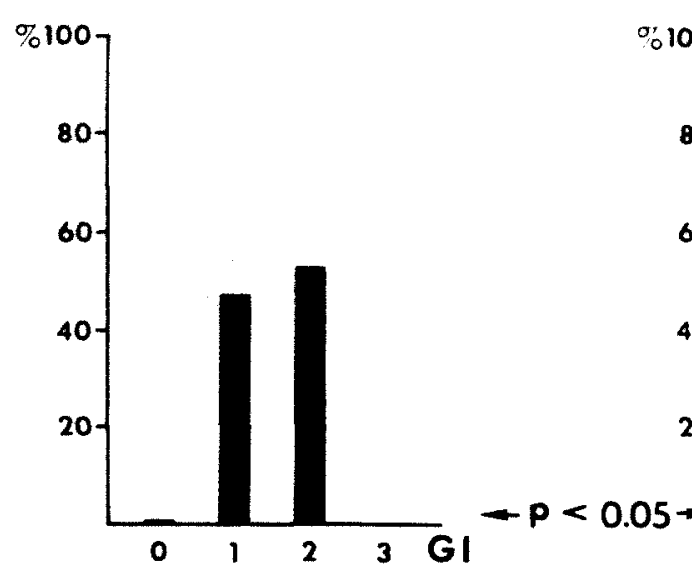

1 YEAR

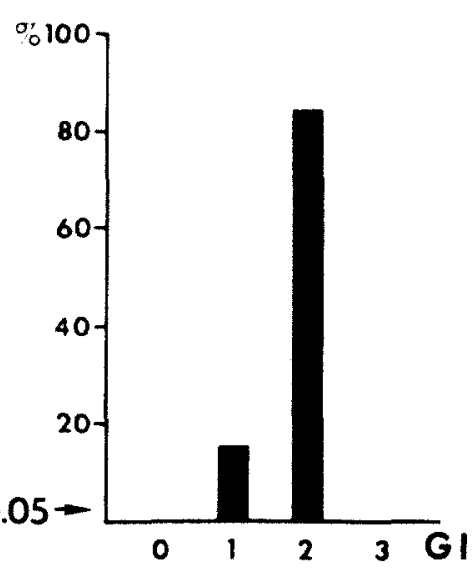

3 YEARS

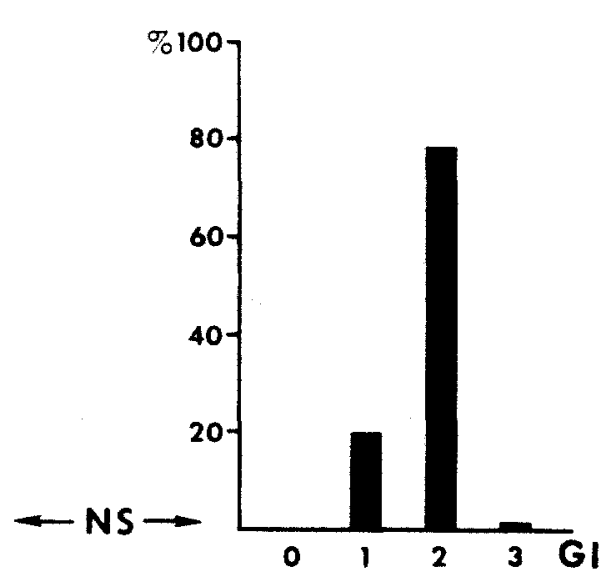

6 YEARS

Fig. 2. Distribution of Gingival Index Scores in Beagle Dogs with Gingivitis*

- Scores were assessed in canines through first molars (C1-M1) of all four quadrants and analyzed by the Median Test. Five animals in each group were used.

plaque of all animals (Table 2). The protein concentrations varied in both supragingival and subgingival plaques The spirochete counts were too low (range $0-10$ per 400 squares of the PHC) to make an accurate quantitative evaluation, and thus the results are given in terms of presence or absence of spirochetes (Table 2). In supragingival plaque spirochetes were found in 2 out of 5 samples from 1-year-old animals, in 3 out of 5 samples from the 6-year-old dogs and in all 5 samples from the 3-yearold dogs (Table 2). In subgingival plaque they were found in only 1 out of 5 samples from the 1-year-old dogs and not detected in any of the samples from the 3 and 6year-old dogs (Table 2).

\section{Table 1}

Sulcus depth and loss of attachment in beagle dogs with gingivitis*

\begin{tabular}{lcc}
\hline Age of dogs & $\begin{array}{c}\text { Sulcus Depth } \\
\text { in } \mathrm{mm}\end{array}$ & $\begin{array}{c}\text { Loss of } \\
\text { Attachment } \\
\text { in mm }\end{array}$ \\
\hline & $\overline{\mathrm{x}} \quad \mathrm{SE}$ & $\overline{\mathrm{x}} \quad \mathrm{SE}$ \\
1 year & $1.50 \pm 0.17$ & $0.00 \pm 0.00$ \\
3 years & $1.02 \pm 0.14$ & $0.03 \pm 0.02$ \\
6 years & $1.27 \pm 0.15$ & $0.04 \pm 0.02$ \\
\hline
\end{tabular}

* Five animals in each group were used.
The number of viable $\mathrm{CFU}$, which were calculated from the anaerobic recovery of the organisms of all dogs, were significantly higher $(p=0.0006)$ in the supragingival samples (Table 4). The numbers of viable CFU in subgingival plaque in the 1 and 6year-old dogs were approximately seven times higher than in the 3-year-old dogs (Table 2). The anaerobic to aerobic ratio was similar in supra and subgingival plaque and did not change with age. A predominately anaerobic flora was found in all plaque samples.

\section{Taxonomic Groups}

When possible, the various bacterial types were placed into different taxonomic groups (Table 3). Streptococci and actinomycetes were the major fermentative organisms in these plaques. Streptococci capable of producing glucans or fructans from sucrose e.g. Streptococcus mutans, Streptococcus sanguis, Streptococcus mitior or Streptococcus salivarius could not be detected. Other streptococci found in the plaques were negative for the following tests: catalase, nitrate reduction, indole and gelatin hydrolysis. Two groups of streptococci were found. Those streptococci which grew on bile- 
Table 2

Plaque parameters of beagle dogs with gingivitis*

\begin{tabular}{lcccccc}
\hline & \multicolumn{3}{c}{ Supragingival Plaque } & \multicolumn{3}{c}{ Subgingival Plaque } \\
\hline Age of Dogs (years) & 1 & 3 & 6 & 1 & 3 & 6 \\
Protein $(\mu \mathrm{g}$ ) per sample & $87.3 \pm 31.5$ & $63.8 \pm 21.2$ & $81.0 \pm 45.8$ & $63.0 \pm 21.9$ & $45.8 \pm 24.2$ & $43.7 \pm 28.9$ \\
Viable CFU per sample $\times 10^{\circ}$ & $29.1 \pm 11.5$ & $18.0 \pm 6.4$ & $18.0 \pm 7.3$ & $0.3 \pm 0.1$ & $0.03 \pm 0.01$ & $0.2 \pm 0.1$ \\
Anaerobic/Aerobic ratio** per sample & $9.2 \pm 4.5$ & $3.9 \pm 1.2$ & $7.2 \pm 2.7$ & $4.7 \pm 2.5$ & $6.7 \pm 3.1$ & $11.6 \pm 4.8$ \\
Spirochete Prevalence & $2 / 5$ & $5 / 5$ & $3 / 5$ & $1 / 5$ & ND & ND \\
\hline
\end{tabular}

- Five dogs in each age group were used. With the exception of spirochetes, the data are expressed as mean values \pm standard error of the means. Sampling site was $\mathrm{p}^{3}$ buccal. ND $=$ Not detectable

** Anaerobic/Aerobic Ratio $=\frac{\text { All CFU recovered anaerobically }}{\text { All CFU recovered aerobically }}$

esculin agar and hydrolyzed esculin (Esculin positive) were placed in Group I. This group probably contained Streptococcus faecalis, enterococci and other non-enterococcus Group D streptococci. Group II was composed of a heterogenous group of streptococci which had one characteristic in common, i.e. they did not hydrolyze esculin. They ranged from aerobic to microaerophilic or anaerobic streptococci and most likely included glucan-negative Streptococcus mitior (S. mitis). Since detailed differentiating physiological or serological tests were not performed, they were not speciated.

The actinomycetes were grouped into five species (Table 3). No actinomycetes except $A$. viscosus produced catalase. With the exception of $A$. bovis the various Actinomyces species were capable of reducing nitrate to nitrite and gave negative starch hydrolysis test. The unclassified actinomycetes-like organisms gave variable results for nitrate reduction, esculin or starch hydrolysis, growth in media and glucose fermentation tests. As they were unidentifiable due to lack of complete data, they would most probably include Actinomyces species and possibly members of other genera.

In addition to actinomycetes, Gram positive, non-spore forming, non-motile, non-fermentative or weakly fermentative filament- ious anaerobic organisms with or without cross septa were found in the plaques in low numbers. They produced trace amounts of acetate and succinate and were identifiable on the basis of esculin hydrolysis test. $P$. acnes was rarely found in the samples. $F$. nucleatum isolates ranged from non-fermentative to weakly fermentative bacteria which gave negative tests for catalase, nitrate reduction, gelatin, starch and esculin hydrolyses.

The pigmented anaerobic bacilli identified as $B$. asaccharolyticus ( $B$. melaninogenicus ss. asaccharolyticus) were tolerant to oxygen (room atmosphere) and exhibited catalase activity. The colonies of these organisms remained viable for 24 to 48 hours on the ETSA plates kept in aerobic atmosphere and could be revived under anaerobic conditions.

Other Gram negative, long, thin rods as well as agar corroding or non-corroding coccobacilli were strictly anaerobic, fastidious organisms. Most of these organisms could not be cultivated beyond primary isolation. Some grew poorly and gave negative results for all criteria that were used. Their growth in broth media was minimal and they formed thin hazy colonies on the agar media. The nitrate reduction test was variable for these organisms. Acetate was the only product that could be detected in the 
Table 3

Predominant cultivable flora of beagle dogs with gingivitis

( $\%$ proportions of cultivable flora)

\begin{tabular}{|c|c|c|c|c|c|c|}
\hline \multirow[b]{2}{*}{ Dogs age (years) } & \multicolumn{3}{|c|}{ Supragingival Plaque } & \multicolumn{3}{|c|}{ Subgingival Plaque } \\
\hline & 1 & 3 & 6 & 1 & 3 & 6 \\
\hline$N=15$ & $\bar{X} \pm S E$ & $\bar{X} \pm S E$ & $\bar{X} \pm S E$ & $\bar{X} \pm S E$ & $\bar{X} \pm S E$ & $\bar{X} \pm S E$ \\
\hline Total Gram Positive Organisms: & $53.7 \pm 12.4$ & $39.9 \pm 10.7$ & $43.0 \pm 13.3$ & $41.0 \pm 13.8$ & $21.9 \pm 9.5$ & $30.2 \pm 4.7$ \\
\hline \multicolumn{7}{|l|}{ Streptococcus: } \\
\hline Strep. spp. (Bile-esculin positive) & $8.0 \pm 4.5$ & $3.6 \pm 1.3$ & $2.0 \pm 1.2$ & $0.8 \pm 0.8$ & $2.1 \pm 1.4$ & $0.6 \pm 0.6$ \\
\hline Strep. spp. (Bile-esculin negative) & $2.8 \pm 1.6$ & $3.6 \pm 2.1$ & $5.4 \pm 2.5$ & $2.3 \pm 2.0$ & $2.1 \pm 0.8$ & $2.8 \pm 1.9$ \\
\hline Actinomyces: & $37.0 \pm 10.7$ & $20.9 \pm 10.5$ & $17.7 \pm 7.8$ & $29.1 \pm 12.2$ & $10.2 \pm 7.0$ & $14.9 \pm 5.1$ \\
\hline A. viscosus & $5.2 \pm 2.5$ & $2.6 \pm 1.8$ & $3.2 \pm 1.4$ & $* 17.2 \pm 7.6$ & $1.5 \pm 0.8$ & $2.2 \pm 0.8$ \\
\hline A. odontolyticus & $8.0 \pm 2.8$ & $5.9 \pm 2.9$ & $3.3 \pm 2.3$ & $3.0 \pm 3.0$ & $0.8 \pm 0.8$ & $1.6 \pm 0.9$ \\
\hline A. israelii & $8.8 \pm 4.0$ & $1.8 \pm 1.3$ & $2.2 \pm 1.2$ & $2.0 \pm 1.3$ & $0.8 \pm 0.8$ & $\begin{array}{c}\text { Not } \\
\text { detected }\end{array}$ \\
\hline A. bovis & $0.6 \pm 0.5$ & $3.8 \pm 1.7$ & $1.2 \pm 0.9$ & $0.8 \pm 0.8$ & $2.5 \pm 2.5$ & $3.0 \pm 2.2$ \\
\hline A. naes/undii & $5.1 \pm 2.7$ & $1.4 \pm 0.9$ & $1.3 \pm 0.4$ & $0.7 \pm 0.4$ & $2.2 \pm 1.6$ & $2.2 \pm 1.4$ \\
\hline Unidentified Actinomycetes & $9.3 \pm 3.7$ & $5.4 \pm 2.9$ & $6.5 \pm 3.7$ & $5.4 \pm 3.3$ & $2.4 \pm 0.9$ & $5.9 \pm 1.3$ \\
\hline \multicolumn{7}{|l|}{$\begin{array}{l}\text { Gram positive rods: } \\
\text { (Anaerobic, Non-fermentative) }\end{array}$} \\
\hline Type I (Esculin+) & $2.3 \pm 0.7$ & $4.5 \pm 1.8$ & $5.9 \pm 1.8$ & $0.3 \pm 0.3$ & $1.9 \pm 1.9$ & $1.2 \pm 1.0$ \\
\hline Type II (Esculin-) & $3.7 \pm 2.7$ & $7.3 \pm 1.1$ & $8.9 \pm 2.8$ & $8.5 \pm 4.6$ & $5.6 \pm 1.9$ & $10.3 \pm 3.3$ \\
\hline Propionibacterium acnes & $\begin{array}{c}\text { Not } \\
\text { detected }\end{array}$ & $\begin{array}{c}\text { Not } \\
\text { detected }\end{array}$ & $3.1 \pm 3.1$ & $\begin{array}{c}\text { Not } \\
\text { detected }\end{array}$ & $\begin{array}{c}\text { Not } \\
\text { detected }\end{array}$ & $0.4 \pm 0.4$ \\
\hline Total Gram Negative Anaerobes: & $41.8 \pm 12.4$ & $53.3 \pm 10.1$ & $52.0 \pm 4.0$ & $50.0 \pm 11.4$ & $69.6 \pm 9.8$ & $58.5 \pm 6.7$ \\
\hline B asaccharolyticus & $16.3 \pm 14.0$ & $29.0 \pm 9.5$ & $27.2 \pm 11.7$ & $1.7 \pm 1.1$ & $23.6 \pm 12.6$ & $16.7 \pm 14.5$ \\
\hline F. nucleatum & $10.8 \pm 6.8$ & $3.4 \pm 1.2$ & $5.4 \pm 3.0$ & $29.8 \pm 8.8$ & $27.1 \pm 10.7$ & $16.2 \pm 5.9$ \\
\hline Gram negative coccobacilli & $6.4 \pm 1.8$ & $9.8 \pm 5.8$ & $10.8 \pm 2.6$ & $10.4 \pm 5.9$ & $15.2 \pm 9.8$ & $21.5 \pm 7.7$ \\
\hline $\begin{array}{l}\text { Gram negative rods: } \\
\text { (Non-fermentative) }\end{array}$ & $8.3 \pm 1.9$ & $11.1 \pm 5.9$ & $8.6 \pm 3.0$ & $8.1 \pm 3.0$ & $3.7 \pm 2.3$ & $4.1 \pm 3.0$ \\
\hline$\%$ Recovery of Organisms & $95.5 \pm 2.7$ & $93.2 \pm 2.1$ & $95.0 \pm 1.7$ & $91.0 \pm 5.5$ & $91.5 \pm 2.7$ & $88.7 \pm 3.4$ \\
\hline
\end{tabular}

* Value significantly higher than the corresponding values in 3 and 6 -year-old dogs $(p=0.05)$.

spent cultures of these coccobacilli. These organisms would probably represent $B$. corrodens and species of the genera Fusobacterium and Bacteroides.

\section{Supragingival Plaque Flora}

The proportions of the different bacterial species were compared between the three age groups (Table 3). Gram positive organisms were found in higher proportions in supragingival plaque of the 1-year-old dogs than in the older animals $(53.7 \%$ vs. $39.9 \%$ and $43.0 \%$; NS) which was explained mainly by the high percentages of actinomycetes and streptococci.

The proportions of Gram negative anaer- obic bacteria were lower in supragingival plaque of the 1-year-old dogs than in the 3 and 6-year-old dogs (41.8\% vs. $53.3 \%$ and $52.0 \%$; NS). B. asaccharolyticus and $F$. nucleatum were predominant in all samples. One of the 1-year-old dogs had an exceptionally high percentage $(72 \%)$ of $B$. asaccharolyticus. If this $\operatorname{dog}$ is excluded from the group, the mean proportions of $B$. asaccharolyticus in the group of older dogs (29.0\% and $27.2 \%$ ) would be 11-12 times higher than in 1-year-old dogs $(2.4 \%)$.

The mean proportions of $F$. nucleatum were higher in the 1-year-old dogs $(10.8 \%)$ than in the older animals (3.4\% and $5.4 \%$; NS). The three age groups had similar pro- 


\section{Table 4}

Comparison of viable counts and proportions of bacteria in the supragingival and subgingival plaques of 15 beagle dogs* with gingivitis

\begin{tabular}{|c|c|c|c|c|c|c|}
\hline & \multicolumn{2}{|c|}{ Supragingival Plaque } & \multicolumn{2}{|c|}{ Subgingival Plaque } & \multicolumn{2}{|c|}{$\begin{array}{c}\text { Significance (p Value) } \\
\text { (Supra vs subgingival } \\
\text { plaque) }\end{array}$} \\
\hline & $\begin{array}{l}\text { Viable } \\
\text { Counts } \\
\times 10^{6} \\
\bar{X} \pm S E\end{array}$ & $\begin{array}{l}\% \text { Pro- } \\
\text { portions } \\
\bar{X} \pm S E\end{array}$ & $\begin{array}{l}\text { Viable } \\
\text { Counts } \\
\times 10^{6} \\
\bar{X} \pm S E\end{array}$ & $\begin{array}{l}\% \text { Pro- } \\
\text { portions } \\
\bar{X} \pm S E\end{array}$ & $\begin{array}{l}\text { Viable } \\
\text { Counts }\end{array}$ & $\begin{array}{l}\% \text { Pro- } \\
\text { portions }\end{array}$ \\
\hline Tota! Viable Bacteria (CFU) & $21.0 \pm 4.7$ & & $0.16 \pm 0.06$ & & 0.0006 & \\
\hline Total Gram Positive Bacteria & $8.1 \pm 2.6$ & $45.2 \pm 6.6$ & $0.06 \pm 0.02$ & $30.6 \pm 6.1$ & 0.006 & 0.05 \\
\hline Total Gram Negative Bacteria & $11.9 \pm 3.5$ & $49.3 \pm 6.6$ & $0.11 \pm 0.04$ & $60.2 \pm 5.8$ & 0.006 & NS \\
\hline Strep. Spp. (Bile-esculin positive) & $1.2 \pm 0.6$ & $4.5 \pm 1.6$ & $0.001 \pm 0.0008$ & $1.3 \pm 0.6$ & NS & 0.05 \\
\hline Strep. Spp. (Bile-esculin negative) & $0.5 \pm 0.2$ & $3.9 \pm 1.2$ & $0.004 \pm 0.002$ & $2.4 \pm 0.8$ & 0.01 & NS \\
\hline Total Actinomycetes & $4.2 \pm 1.6$ & $24.9 \pm 5.7$ & $0.04 \pm 0.02$ & $17.8 \pm 5.2$ & 0.02 & NS \\
\hline A. viscosus & $0.4 \pm 0.2$ & $3.6 \pm 1.1$ & $0.02 \pm 0.01$ & $6.9 \pm 3.1$ & 0.02 & NS \\
\hline A. odontolyticus & $0.9 \pm 0.3$ & $5.7 \pm 1.6$ & $0.004 \pm 0.003$ & $1.8 \pm 1.0$ & 0.01 & 0.03 \\
\hline A. israelii & $1.0 \pm 0.6$ & $4.1 \pm 1.5$ & $0.003 \pm 0.002$ & $1.0 \pm 0.5$ & NS & NS \\
\hline A. bovis & $0.3 \pm 0.2$ & $2.0 \pm 0.8$ & $0.002 \pm 0.001$ & $2.1 \pm 1.1$ & NS & NS \\
\hline A. naes/undii & $0.5 \pm 0.4$ & $2.5 \pm 1.0$ & $0.002 \pm 0.001$ & $1.7 \pm 0.8$ & NS & NS \\
\hline $\begin{array}{l}\text { Unidentified actinomycetes } \\
\text { Type I Gram Positive Rods }\end{array}$ & $1.1 \pm 0.6$ & $7.0 \pm 1.9$ & $0.008 \pm 0.005$ & $4.3 \pm 1.2$ & NS & NS \\
\hline $\begin{array}{l}\text { (Esculin }+ \text { ) } \\
\text { Type II Gram Positive Rods }\end{array}$ & $0.9 \pm 0.3$ & $4.3 \pm 0.9$ & $0.001 \pm 0.0007$ & $1.2 \pm 0.8$ & 0.01 & 0.04 \\
\hline (Esculin -) & $1.0 \pm 0.3$ & $6.6 \pm 1.3$ & $0.01 \pm 0.005$ & $7.8 \pm 1.8$ & 0.002 & NS \\
\hline Propionibacterium acnes & $0.3 \pm 0.4$ & $1.0 \pm 1.0$ & $0.007 \pm 0.0007$ & $0.1 \pm 0.1$ & NS & NS \\
\hline B. asaccharolyticus & $6.9 \pm 2.9$ & $24.5 \pm 6.4$ & $0.007 \pm 0.003$ & $14.5 \pm 6.4$ & 0.03 & NS \\
\hline F. nucleatum & $1.6 \pm 1.0$ & $6.4 \pm 2.3$ & $0.06 \pm 0.03$ & $25.1 \pm 5.3$ & NS & 0.006 \\
\hline $\begin{array}{l}\text { Gram Negative Coccobacilli } \\
\text { Gram Negative Bacilli }\end{array}$ & $1.5 \pm 0.5$ & $9.0 \pm 2.3$ & $0.03 \pm 0.01$ & $15.3 \pm 4.6$ & 0.02 & NS \\
\hline $\begin{array}{l}\text { (non-fermentative) } \\
\% \text { Recovery of the Cultivable }\end{array}$ & $1.9 \pm 0.6$ & $9.4 \pm 2.3$ & $0.01 \pm 0.007$ & $5.3 \pm 1.6$ & 0.01 & NS \\
\hline Organisms & & $94.5 \pm 1.2$ & & $90.8 \pm 0.02$ & & 0.02 \\
\hline
\end{tabular}

* Determined by paried t-test.

portions of non-pigmented coccobacilli $(6.4 \%, 9.8 \%$ and $10.8 \%)$ as well as long, thin, Gram negative, non-fermentative rods $(8.3 \%, 11.1 \%$ and $8.6 \%)$.

\section{Subgingival Plaque Flora}

Gram negative organisms predominated in the subgingival flora of these dogs (Table 3). Some organisms exhibited poor growth and could not be characterized. The mean proportions of Gram negative organisms in subgingival plaque were similar in the 1 and 6-year-old animals (50\% and 58.5\%) and slightly but insignificantly higher in the 3year-old dogs $(69.6 \%)$. The prevalence of these organisms was also considered on individual species basis. Twice as many $F$. nucleatum were found in the 1 and 3-yearold animals as in the 6-year-old dogs (NS, Table 3). The proportions of $B$. asaccharolyticus in subgingival plaque of the 1-yearold dogs were approximately 14 times lower than in the 3-year-old animals and 10 times lower than in the 6-year-old dogs. The proportions of $B$. asaccharolyticus were lower in subgingival than in supragingival plaque. In this regard, the proportions of $F$. nucleatum and anaerobic Gram negative coccobacilli were higher in subgingival than in supragingival plaque (NS). However, due to 
the wide variations within each age group, the proportions of $B$. asaccharolyticus and $F$. nucleatum did not differ significantly between the age groups (Table 3).

When subgingival plaques were analyzed with respect to Gram positive organisms, their proportions in the 1-year-old dogs were higher than in the 3 or 6-year-old dogs $(41.0 \%$ vs $21.9 \%$ in 3 and $30.2 \%$ in 6year-old dogs, NS). Two to three times as many actinomycetes were found in the 1 year-old animals as in the older dogs. $A$. viscosus was the most prominent bacterial species in subgingival plaque of the 1-yearold dogs, its proportions being 8-11 times higher than in the older animals $(17.2 \%$ vs. $1.51 \%$ and $2.2 \% \mathrm{p}=0.05$ ).

Streptococci and total Gram negative organisms were comparable in all three age groups. Veillonella, Selenomonas, Campylobacter or other motile rods and fermentative, pigmented bacteroides, e.g. B. melaninogenicus ss. intermedius were not observed in any of the plaque samples studies.

\section{Combined Data Analysis of Plaque Flora}

The data from the three groups of dogs were combined irrespective of their age and compared to determine whether the differences between supragingival and subgingival plaques were significant in regard to the proportions of various organisms and total viable counts. The results are summarized in Table 4. Supragingival plaques had significantly higher total viable CFU $(21.5 \times$ $10^{6}$ vs. $\left.0.16 \times 10^{6} ; \mathrm{p}=0.0006\right)$ including the total Gram positive organisms $(8.1 \times$ $10^{6}$ vs. $0.06 \times 10^{6} ; \mathrm{p}=0.006$ ), the total Gram negative organisms $\left(11.9 \times 10^{6}\right.$ vs. $\left.0.11 \times 10^{6} ; \mathrm{p}=0.006\right)$ and the total actinomycetes CFU $\left(4.2 \times 10^{6}\right.$ vs. $0.04 \times 10^{6}$; $\mathrm{p}=0.02$ ). Comparison of the viable CFU of various bacterial species in the two types of plaques showed that bile-esculin negative streptococci, A. viscosus, A. odontolyticus, Gram positive filamentous rods, $B$. asac- charolyticus, Gram negative coccobacilli and Gram negative bacilli CFU were significantly higher in supragingival plaque (Table 4).

When the proportions of the organisms were compared, the Gram positive bacteria were significantly higher in the supragingival plaque (Table 4). Although the proportions of Gram negative bacteria were higher in the subgingival plaque as compared to the supragingival plaque, $(60.2 \%$ vs. $49.3 \%$ ) the differences were not significant. When the proportions of individual bacterial species were compared, $F$. nucleatum was the only organism found in significantly higher proportions in subgingival plaque $(25.1 \%$ vs. $6.4 \%, \mathrm{p}=0.006)$. The proportions of bile-esculin positive Streptococcus sp., $A$. odontolyticus and esculin positive, filamentous, Gram positive rods were significantly higher in supragingival plaque (Table 4).

\section{Discussion}

The qualitative and quantitative composition of the dominant cultivable plaque flora was determined in beagle dogs with gingivitis but with no or insignificant loss of connective tissue attachment. Organisms which were uncultivable by the culturing technique used, and the ones that were lost during the primary isolation period, were not included. In order to reduce site to site variability, the same investigator removed supra- and subgingival plaques from the same tooth in all animals. One, three and six-year old dogs were used to explore whether the age of the dogs had any impact on the composition of the plaque flora. Although the proportions of the organisms showed an increase or decrease with age*.

* Only the proportions of $A$. viscosus in subgingival plaque showed a significant change with age i.e. a decrease. 
The results based either on individual age groups or combined data analyses showed that both supra- and subgingival plaques had a predominantly anaerobic flora with high proportions of $B$. asaccharolyticus and $F$. nucleatum. Supragingival plaque had higher proportions of Gram positive organisms $(p=0.05)$, significantly higher viable counts $(\mathrm{p}=0.0006)$ and a higher frequency of spirochetes than the subgingival plaque.

The presence of spirochetes in 10 of 15 supragingival and in only 1 of 15 subgingival samples indicates that proper precautions were taken during the sampling procedure to avoid contamination of the subwith the supragingival sample. Also, the recovery of high proportions of $F$. nucleatum and $B$. asaccharolyticus and other anaerobes from the two types of plaque demonstrates the adequacy of the plaque collection procedure and attests to the suitability of prereduced transport and culture media, plaque dispersion procedure and the anaerobic technique, that were used.

The superiority of continuous anaerobic procedures in recovering more varieties of bacteria from the nose, throat and fecal flora of beagles was reported by Balish et al. (1977), who were able to isolate and identify more species of anaerobes with the glove box technique than when using Gas Pak anaerabic jars (Baltimore Biological Laboratories, Cockeysville, Md.). In our study, we were able to isolate fastidious anaerobes which could not be subcultured beyond the primary isolation stage due most likely to the absence of their growth requirements in our media. That also may be the reason why we could not culture the spirochetes that were found microscopically in the specimens.

A fluorometric technique was used for determining the micro-quantities of plaque protein under the assumption that the protein would represent the bacterial mass and accordingly could serve as a reference for the bacteriological data. Since the plaque quantity was small in some cases, and the protein was beyond detectable limits, a high variability in protein concentration was seen among the samples. Also, the inclusion of nonbacterial protein might have increased some of the values.

Sonication has been used for the dispersion of plaque (Syed \& Loesche 1972, Manganiello et al. 1977). Pure cultures of Gram negative bacteria seem to be more sensitive to such treatment than pure cultures of Gram positive organisms (Robrish et al. 1976). Experiments with a micro-ultrasonic cell disrupter (Kontes, Vineland, N.J.) showed that a 20 second sonication period did not lyse the Gram negative species or favor a high recovery of Gram positive organisms (Syed \& Loesche 1978b), which is in agreement with the results presented in Table 3.

Supra- as well as subgingival plaques were predominantly composed of anaerobes. The low numbers of streptococci and the presence of nonfermentative organisms are characteristic of the beagle flora and makes it different from human dental plaque, which is in keeping with findings reported by Wunder et al. (1976). Another difference from human dental plaque was that $S$. sanguis, Veillonella, Selenomonas and Campylobacter were not found in the present study. $S$. mutans could not be detected in any of the animals. $F$. nucleatum and $B$. asaccharolyticus were found in significant proportions in both the supra- and subgingival plaques. $B$. asaccharolyticus was dominant in supragingival plaque and $F$. nucleatum was found in higher proportions in subgingival plaque. Also the beagle dogs harbored unusually high proportions of $B$. asaccharolyticus and $F$. nucleatum in their plaque as compared to what has been found in humans (Gibbons et al. 1963, Loesche, Hockett \& Syed 1972, van Palenstein Helderman 1975, Williams, Pantolone \& Sherris 1976). This is in agreement with findings in the beagle dog re- 
ported by Courant et al. (1968) who found twice the percentage of $B$. melaninogenicus seen in human plaque. Also, the canine strains of $B$. asaccharolyticus displayed catalase activity and survived for 1 to 2 days in aerobic atmosphere. Serological tests with fluoresein conjugated antisera against human $B$. asaccharolyticus were negative for the canine strains (E. Grenier, personal communication). Catalase activity, increased tolerance to oxygen (aerobic atmosphere) and serological dissimilarity are the characteristics which would differentiate canine $B$. asaccharolyticus from human strains.

Spirochetes were found in the majority of the supragingival plaque samples but only in 1 of 15 subgingival samples, although Soames and Davies (1975) found a predominance of spirochetes in the crevice of a young beagle dog. Our findings are in agreement with Krasse and Brill (1960), who found spirochetes in only $15 \%$ of subgingival plaque samples in dogs and Courant et al. (1968), who did not observe any spirochetes in microscopic preparations of subgingival debris of beagle dogs. This would suggest that these organisms were either nonexistant in these sites or present in too small numbers to be detectable by the light microscopic techniques. Another group of fusiform shaped, microaerophilic, gliding organisms, which are presently classified as B. ochraceus (Bergey's Manual 1974, Capnocytophaga) could not be detected as one of the predominant organisms in plaque of the beagle dog.

The present study was designed to examine the composition of supra- and subgingival plaque in beagle dogs with established gingivitis. The predominant bacterial groups were identified. The prevalence of hitherto unknown and uncultivable organisms remains to be determined. However, the results of this study might serve as the basis for future investigations concerning the taxonomy and the periodontal disease as- sociated plaque microbiology in the beagle dogs.

\section{Acknowledgements}

The encouragement by Dr. Walter Loesche in the initiation of this study and his criticism during the preparation of this manuscript is gratefully acknowledged. Janice Stoll, Dennis Ritter and Byron Scott assisted in this study. This investigation was supported by grant $\mathrm{DE}-02731$ from the $\mathrm{Na}$ tional Institute of Dental Research, Bethesda, Maryland, U.S.A.

\section{References}

Aranki, A., Syed, S. A., Kenney, E. B. \& Freter, R. 1969. Isolation of anaerobic bacteria from human gingiva and mouse cecum by means of simplified anaerobic glove box procedure. Applied Microbiology 17: 568-576.

Balish, E., Cleven, D., Browen, J. \& Yale, C. E. 1977. Nose, throat and fecal flora of beagle dogs housed in locked or open environments. Applied Environmental Microbiology 34: 207-211.

Buchanan, R. E. \& Gibbons, N. E. (ed.) 1974. Bergey's Manual of Determinative Bacterio$\log y, 8$ th ed. Baltimore, Maryland: Williams and Wilkins Co.

Courant, D. R., Saxe, S. R., Nash, L. \& Roddy, S. 1968. Sulcular bacteria in the beagle dog. Periodontics 6: 250-252.

Finegold, S .M. \& Barnes, E. M. 1977. Report of the ICBS Taxonomic Subcommittee on Gram Negative Anaerobic Rods. Proposal that the saccharolytic and asaccharolyticus strains at present classified in the species Bacteroides melaninogenicus (Oliver and Wherry) be reclassified into two species Bacteroides melaninogenicus and Bacteroides asaccharolyticus. International Journal of Systematic Bacteriology 27: 388-391.

Gibbons, R. J., Socransky, S. S., Sawyer, S., Kapsimalis, B. \& MacDonald, J. B. 1963. The microbiota of the gingival crevice area of man. II. The predominant cultivable organisms. Archives of Oral Biology 8: 281289.

Hamp, S. E., Lindhe, J. \& Heyden, G. 1972. Experimental gingivitis in the dog. An en- 
zyme histochemical study. Archives of Oral Biology 17: 329-337.

Hamp, S. E., Lindhe, J. \& Löe, H. 1973. Long term effects of chlorhexidine on developing gingivitis in the beagle dog. Journal of Periodontal Research 8: 63-70.

Holdeman, L. V. \& Moore, W. E. C. (ed). 1975. Anaerobe Laboratory Manual. Blacksburg, VA.: Virginia Polytechnique Institute and State University.

Karnovsky, M. J. 1965. A formaldehydeglutaraldehyde fixative of high osmolarity for use in electron microscopy. Journal of Cell Biology 27: 137A-138A.

Krasse, B. \& Brill, N. 1960. Effect of consistency of diet on bacteria in gingival pocket in dogs. Odontologisk Revy 11: 152-165.

Lindhe, J., Hamp, S. E. \& Löe, H. 1973. Experimental periodontitis in the beagle dog. Journal of Periodontal Research 8: 1-10.

Lindhe, J., Hamp, S. E. \& Löe, H. 1975. Plaque induced periodontal disease in beagle dogs - a 4 year clinical, roentgenographical and histometric study. Journal of Periodontal Research 10: 243-255.

Löe, H. \& Silness, J. 1963. Periodontal disease in pregnancy. I. Prevalance and severity. Acta Odontologica Scandinavica 21: 533-551.

Loesche, W. J., Hockett, R. N. \& Syed, S. A. 1972. The predominant cultivable flora of tooth surface plaque removed from institutionalized subjects. Archives of Oral Biology 17: 1311-1328.

Luft, J. H. 1961. Improvements in epoxy resin embedding methods. Journal of Biophysical and Biochemical Cytology 9: 409-414.

Luna, L. G. (ed). 1968. Manual of Histologic Staining Methods o fthe Armed Forces Institute of Pathology 3rd Edition. New York: McGraw-Hill Book Company.

Manganiello, A. D., Socransky, S. S., Smith, C., Propas, D., Oram, V. \& Dogon, I. L. 1977. Attempts to increase viable count recovery of human supragingival dental plaque. Journal of Periodontal Research 12: 107-119.

Minah, G. \& Loesche, W. J. 1977. Sucrose metabolism in resting cell suspensions of caries-associated and non-caries-associated dental plaque. Infection and Immunity 17: 43-54.

Newman, M. J., Sander, M., Oremerod, W., Angel, L. \& Goldhaber, P. 1977. The effect of dietary gantrisin supplements on the flora of periodontal pockets in four beagle dogs. Journal of Periodontal Research 12: 129-134. Robrish, S. A., Grove, S. B., Bernstein, R. S.,
Marucha, P. T., Socransky, S. S. \& Amdur, B. 1976. Effect of sonic treatment on pure cultures and aggregates of bacteria. Journal of Clinical Microbiology 3: 474-479.

Saxe, S. R., Green, J. C., Bohanan, H. M. \& Vermillion, J. R. 1967. Oral debris calculus, and periodontal disease in the beagle dog. Periodontics 5: 217-225.

Saphir, D. A. \& Carter, G. R. 1976. Gingival flora of the dog with special reference to bacteria associated with bites. Journal of Clinical Microbiology 3: 344-349.

Schroeder, H. C., Graf-De-Beer, M. \& Attstrøm, R. 1975. Initial gingivitis in dogs. Journal of Periodontal Research 10: 128-142.

Schroeder, H. E., Lindhe, J., Hugoson, A. \& Munzel-Pedrazzoli, S. 1973. Structural constitutents of clinically normal and slightly inflamed dog gingiva. A morphometric study. Helvetica Odontologica Acta 17: 70-83.

Schroeder, H. E. \& Lindhe, J. 1975. Conversion of established gingivitis in the dog into destructive periodontitis. Archives of Oral Biology 20: 775-782.

Silness, J. \& Löe, H. 1964. Periodontal disease in pregnancy. II. Correlation between oral hygiene and periodontal condition. Acta Odontologica Scandinavica 22: 121-135.

Soames, J. V. \& Davies, R. M. 1975. The structure of subgingival plaque in a beagle dog. Journal of Periodontal Research 9: 333-341.

Socransky, S. S., Gibbons, R. J., Dale, A. C., Bortnick, L., Rosenthal, E. \& MacDonald, J. B. 1963. The microbiota of the gingival crevice area of man. I. Total microscopic and viable counts of specific organisms. Archives of Oral Biology 8: 275-280.

Syed, S. A. 1976. A new medium for the detection of gelatin hydrolyzing activity of human dental plaque flora. Journal of Clinical Microbiology 3: 200-202.

Syed, S. A. \& Loesche, W. J. 1972. Survival of human dental plaque flora in various transport media. Applied Microbiology 24: 638644.

Syed, S. A. \& Loesche, W. J. 1978a. Bacteriology of human experimental gingivitis. I. Effect of plaque age. Infection and Immunity 21: 821-829.

Syed, S. A. \& Loesche, W. J. 1978b. Efficiency of Kontes ultrasonic cell disruptor in the dispersion of dental plaque and pure cultures of oral flora. Abstract \#982. Journal of Dental Research Special Issue, Volume 57, 1978.

van Palenstein Helderman, W. H. 1975. Total viable count and differential count of Vibrio 
(Campylobacteria) sputorum, Fusobacterium nucleatum, Selenomonas sputigena, Bacteroides ochraceus and Veillonella in the inflamed and heathy gingival crevice. Journal of Periodontal Research 10: 294-306.

Weigele, M., DeBernardo, S. L., Tengi, J. P. \& Leimgruber, W. 1972. A novel reagent for the fluorometric assay of primary amines. Journal of American Chemical Society 94: 5927-5928.

Williams, B. L., Pantolone, R. M. \& Sherris, J. C. 1976. Subgingival microflora and periodontitis. Journal of Periodontal Research 11: 1-18.
Wunder, J. A., Briner, W. \& Calkins, G. P. 1976. Identification of the cultivable bacteria in dental plaque from the beagle dog. Journal of Dental Research 55: 1097-1102.

Address:

Department of Oral Biology

School of Dentistry

3204 Dental Building

The University of Michigan

Ann Arbor, Michigan 48109

U.S.A. 
This document is a scanned copy of a printed document. No warranty is given about the accuracy of the copy. Users should refer to the original published version of the material. 